Diabetologia (1991) 34 [Suppl 1]: S8-S10

0012186X9100100X

\title{
Pancreas transplantation in Lyon: overall results
}

\author{
X. Martin ${ }^{1}$, N. Lefrancois ${ }^{2}$, J. M. Marechal ${ }^{1}$, A. Gelet ${ }^{1}$, J. L. Viguier ${ }^{1}$ and J. M. Dubernard ${ }^{1}$ \\ ${ }^{1}$ Department of Urology and Transplantation Surgery, and \\ ${ }^{2}$ Department of Transplantation Medicine, Hôpital E. Herriot, Lyon, France
}

Summary. A total of 182 pancreatic transplantations were performed between 1976 and 1990. Survival of the grafts was $45 \%$ at 5 years. Major causes of graft loss have been rejection and venous thrombosis. Death occured in 24 patients and was usually related to vascular complications. The techniques of duodenopancreatic transplantation with enteric drainage, bladder drainage and the technique of segmental duct obstructed grafts were compared. A higher rate of surgical complications was observed with enteric drainage, whereas it is unclear from the data whether segmental grafts or duodenopancreatic grafts drained into the bladder lead to different results.

Key words: Transplantation-Pancreas-Duct obstructionEnteric drainage- Bladder drainage

\section{Introduction}

A pancreas transplantation programme was strarted in Lyon in 1976 using the technique of segmental grafting with neoprene injection. This technique was originally described by Dubernard et al. (1976) in animals. Exocrine sectretion of the gland is blocked by intra-ductal injection of a synthetic polymer that solidifies when in contact with pancratic secretions. This technique appeared to be very convenient as it eliminates the need for diversion of the pancreatic secretions in another organ which can induce surgical complications. In addition this technique could be associated with segmental harvesting of the gland, thereby simplifying organ removal.

This technique has been used extensively in Lyon and in many other groups in the seventies and early eighties. In fact the need for massive immunosuppression and especially steroid therapy caused many surgical complications such as fistulas when enteric drainage of the pancreatic secretions was used. In the mid eighties cyclosporin A allowed other techniques of enteric drainage to be utilized as well as techniques of bladder drainage of the pancreatic secretion.(Mac Donald 1982).
In our institution, after extensive use of the segmental injection technique until 1986 , we have tested other techniques such as whole pancreas transplantation with enteric drainage, and later, whole organ transplantation with bladder drainage.

Our objective is to compare the different types of techniques in terms of the mass of tissue and the type of management of the exocrine secretion .

\section{Subjects and methods}

Our study involved 182 patients with type 1 diabetes mellitus who had received pancreas transplants. General data concerning characteristics of the patients are summarized in Table 1. Data concerning age, mean duration of diabetes presence of dialysis or ophtalmologic complications were identical in all patients whereas data concerning vascular complications (macroangiopathy, cardiac complications, amputations) showed an improvement of the status of the patients probably due to better care for the diabetic patients and better selection in the latest series.

The technique used for the first 55 patients was segmental transplantation with neoprene injection (from 1976 to 1985). Then we performed a comparative trial between segmental grafts and total duodeno-pancreatic grafts with enteric diversion (33 patients), followed by a series of 33 consecutive segmental injected grafts, and then another comparative series between segmental injected grafts and total duodeno-pancreatic grafts this time drained into the bladder (67 patients).

Immunosuppression varied from 1976 to 1990 and six different protocols were used : Protocol A: azathioprine (2-3 $\left.\mathrm{mg} \cdot \mathrm{kg}^{-1} \cdot \mathrm{day}^{-1}\right)$, prednisone ( $1 \mathrm{mg} \cdot \mathrm{kg}^{-1} \mathrm{day}^{-1}$ for 10 days then decrease), antilymphocyte globulin 4 to 12 weeks ; Protocol B : cyclosporin A $8 \mathrm{mg} \cdot \mathrm{kg}^{-1}$. day ${ }^{-1}$ and antilymphocyte globulin 8 to 12 weeks; Protocol C : identical to protocol A plus cyclosporin starting 4 to 6 weeks post-operativelly ; Protocol D: azathioprine ( 2 to $3 \mathrm{mg} . \mathrm{kg}^{-}$ 1. $\mathrm{day}^{-1}$, Prednisone $0.25 \mathrm{mg} \cdot \mathrm{kg}^{-1} \cdot \mathrm{day}^{-1}$ and cyclosporin (6$\left.8 \mathrm{mg} . \mathrm{kg}^{-1} \cdot \mathrm{day}^{-1}\right)$; Protocol E: azathioprine $\left(2-3 \mathrm{mg} \cdot \mathrm{kg}^{-}\right.$ $1 \cdot \mathrm{day}^{-1}$ ), prednisone $0.25 \mathrm{mg} \cdot \mathrm{kg}^{-1} \cdot \mathrm{day}^{-1}$, cyclosporin 4-6 $\mathrm{mg} \cdot \mathrm{kg}^{-1} \mathrm{day}^{-1}$ and antilymphocyte globulin or OKT3 for 14 
days ; Protocol F : azathioprine $\left(2-3 \mathrm{mg} \cdot \mathrm{kg}^{-1} \cdot \mathrm{day}^{-1}\right)$, prednisone $\left(0.25 \mathrm{mg} \cdot \mathrm{kg}^{-1}\right.$. day $\left.{ }^{-1}\right)$, cyclosporin $\left(4-6 \mathrm{mg} \cdot \mathrm{kg}^{-}\right.$ $1 \cdot \mathrm{day}^{-1}$ ) and antilymphocyte globulin ; Protocol G : azathioprine $\left(2-3 \mathrm{mg} \cdot \mathrm{kg}^{-1} \cdot \mathrm{day}^{-1}\right)$, prednisone $\left(1 \mathrm{mg} \cdot \mathrm{kg}^{-1}\right.$. day $^{-1}$ for 2 weeks then decrease to $10-15 \mathrm{mg}$. day $^{-1}$ ), cyclosporin 4-6 mg $\cdot \mathrm{kg}^{-1} \cdot \mathrm{day}^{-1}$ and antilymphocyte globulin for 14 days. Patients were followed postoperatively and neuropathy, ophtalmologic complications, control of diabetes have been studied and are reported elsewere in this issue.

Results are expressed in terms of surgical complications and graft losses.

\section{Results}

\section{Overall results}

Overall results show that the number of functioning grafts is 74 after 182 performed. Results in terms of the period of transplantation and the immunosuppression are shown in the figure. Graft losses were : rejection (39 cases), venous thrombosis (28 cases) arterial thrombosis(14 cases), or surgical complications such as pancreatitis of the graft, haemorrage ( 3 cases).

Rejection was observed less frequently with improvement of the immunosuppression as shown in Figure 1 . For diagnosis of pancreas rejection, kidney rejection was employed as a marker. The use of urinary amylases in the latest group of patients with bladder drainage was disapointing in this regard.

Venous thrombosis was a major cause of graft loss in our series. The incidence of this complication decreased markedly since 1988, after University of Wisconsin solution was used routinely in our institution (Wahlberg

1987). However the rate of thrombosis was still significant in $1989(3 / 29)$ and in 1990 (3/31). Death was observed in 24 cases. Death was related in most cases to cardiovascular complication such as myocardial infarction or cerebro-vascular obstruction.

\section{Surgical techniques}

Surgical complications according to the technique used were especially studied. When comparing segmental duct obstructed grafts (DO) vs whole organ with enteric drainage (ED), the number of surgical complications requiring re-operation was higher in the group of patients with enteric drainage ( 6 vs 1 ); however the number of graft losses was identical in both groups (6 vs 5 ). The main causes of loss of pancreatic graft function in this comparative trial was : early vascular thrombosis (two grafts lost in DO group and three in ED group) and rejection : one graft was lost from rejection at 8 months in DO group in a patient with irreversible kidney rejection 2 months after transplantation. Two rejection episodes occured in ED group : in the first patient 34 months after transplantation (the kidney was lost from rejection 20 months after double transplantation). In the second patient both organs were rejected 14 months after transplantation
One patient in DO group died with a functioning graft 5 months after transplantation (necrosing enteritis) and two

Table 1. Characteristics of the 182 patients receiving a pancreas transplant in Lyon

\section{6-1985 1985-1990}

$\begin{array}{ccc}\begin{array}{c}\text { Age (years(SD)) } \\ \text { Duration of } \\ \text { diabetes }\end{array} & 37.2(11) & 36.9(7,6) \\ \begin{array}{c}\text { (years(SD)) } \\ \text { Dialysis (n) }\end{array} & 46(2,5) & 23.9(1,6) \\ \text { Dial. Duration } & 10.0(2) & \\ \text { (months( SD)) } & & 111 \\ \text { Retinopathy (n) } & 51 & 16(6) \\ \text { Macroangiopathy } & 40 & 127 \\ \text { Myocardiopathy } & 24 & 31 \\ \text { Neuropathy(n) } & 55 & 38 \\ & & 127\end{array}$

transplantectomies were necessary : one for a major postoperative bleeding in DO group and the other for an extensive pancreatic necrosis probably due to a prolonged cold ischaemia in the ED group. Twelve DO (71\%) and eight ED (57\%) pancreatic grafts are still functioning and all these patients are insulin-independent except one (with DO graft) requiring insulin therapy (30 \% of pretransplant dose) despite a preserved C-peptide secretion. Complications requiring iterative surgery were more frequent in the ED group : one enteric leakage, two small bowel occlusions, one post-operative bleeding and two wound dehisences were observed in the ED group. In the DO group only one wound dehisence and one postoperative bleeding occurred.

Our present results in the trial comparing bladder drainage (BD-25 patients) and segmental duct obstructed (DO-26 patients) grafts are the following : venous thrombosis : 2 in $\mathrm{BD}$ vs 5 respectively ; surgical condition requiring iterative surgery : 2 in $\mathrm{BD}$ vs 1 , wound infection 14 in ED vs 4 , pancreatic fistula 2 vs 2 ; bladder fistula 2 in $B D$ vs 0 ; Urologic complication episodes (Urinary tract infection 65 episodes in $B D$ vs 45 in $E D$, chronic retention 3 in $\mathrm{BD}$ vs 1 in $\mathrm{DO}$, chemical cystitis 2 in $\mathrm{BD}$ ) were significantly more frequent in the BD group. Loss of pancreatic grafts and of kidney grafts were identical in both groups ( 6 and $6 ; 3$ and 2 , respectively)

Actuarial survival rates are at present 61 in the BD group versus 57 in the DO group at 2 years.

\section{Discussion}

Overall survival of pancreas transplants is $45 \%$ at 5 years, most transplant loss occurs during the first 2 years ; global survival does not seem to be influenced by the technique. Function of the grafts is similar whatever the technique used. However more diabetic-type glucose tolerance tests were found in a small group of patients with segmental grafts as compared to total grafts with enteric diversion. (La Rocca 1988). Similar findings were not found in our latest tests in this series or in the series 
of total grafts drained in the bladder. Data from the International Registry of Pancreatic Tansplantation found a difference of graft function according to the technique.

Longer follow-up will determine which technique has the best functional results and the lower rate of surgical complications.

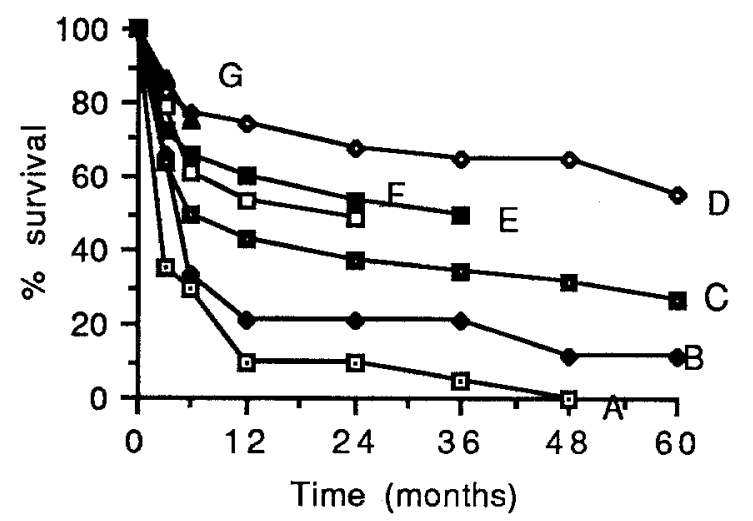

Fig.1. Survival of the pancreatic graft according to the protocol of immunosuppression used (A, B, C, D, E, F and G)

\section{References}

Dubemard JM, Traeger J, Neyra P, Touraine JL, Blanc Brunat N ((1976) A new method of preparation of segmental pancreatic grafts for transplantation. Surg 84:634-639

La Rocca, Secchi A, Dubernard JM, Melandri M, Monti L (1989) Metabolic effects of total versus segmental pancreas allotransplantation. Diabetes 38: (Suppl):259

MacDonald AS, MacKinnon JG, Malatajalian JM (1982) Pancreatic autotransplantation: duct drainage to bladder, duodenum, stomach compared to duct ligation or free drainage into the peritoneal cavity. Transpl Proc 14:705-707

Martin X, Dubemard JM, Sanserverino R, Melandri M, Faure JL, Lefrancois N (1989) Segmental obstructed pancreas grafts versus pancreatico-duodenal grafts with enteric diversion. Diabetes 38 (Suppl):16-17

Prieto M, Sutherland DER, Fernandez Cruz L, Meil J, Najarian D (1987) Experimental and clinical experience with urine amylase monitoring for early diagnosis of rejection in pancreas transplantation. Transplantation 43:73-79

Wahlberg JA, Love R, Landegaard L, Southard JH, Belzer FO (1987) Seventy hours preservation of canine pancreas. Transplantation $43: 5-8$

Dr. X. Martin

Service d'Urologie

Chirurgie de la Transplantation

Hôpital Edouard Herriot

place d'Arsonval

F-69437 Lyon Cedex 03

France 